\title{
Optimal Strength and Number of Shocks at Upper Limit of Vulnerability Testing Required to Predict High Defibrillation Threshold Without Inducing Ventricular Fibrillation
}

\author{
Kaoru Takami, MD; Akihiro Yoshida, MD; Koji Fukuzawa, MD; Asumi Takei, MD; \\ Kunihiko Kiuchi, MD; Gaku Kanda, MD; Hiroyuki Kumagai, MD; Mitsuru Takami, MD; \\ Satoko Torii-Tanaka, MD; Mitsuaki Itoh, MD; Kimitake Imamura, MD; \\ Ryudo Fujiwara, MD; Atsushi Suzuki, MD; Tomoyuki Nakanishi, MD; \\ Soichiro Yamashita, MD; Akinori Matsumoto, MD; Ken-ichi Hirata, MD
}

\begin{abstract}
Background: The upper limit of vulnerability (ULV) closely correlates with the defibrillation threshold (DFT). The aim of this study was to establish the optimal protocol for using the ULV test to predict high DFT (>20 J) without inducing ventricular fibrillation (VF).
\end{abstract}

\begin{abstract}
Methods and Results: The 10-J and 15-J ULV test with 3 coupling intervals $(-20,0$, and +20 ms to the peak of T-wave) and the DFT test were performed in 96 patients receiving implantable cardioverter defibrillator. ULV $\leq 10 \mathrm{~J}$ was confirmed in 47 (49\%). ULV $\leq 15 \mathrm{~J}$ was confirmed in 70 (77\%) of 91 patients (15-J ULV test could not be done in 5). The sensitivity and negative predictive value of both ULV $>10 \mathrm{~J}$ and $>15 \mathrm{~J}$ for predicting high DFT were $100 \%$. The specificity and positive predictive value of ULV >15J were higher than those for ULV >10J (85\% vs. 55\%, 43\% vs. $22 \%$, respectively). The rate of VF inducibility for confirming ULV $\leq 15 \mathrm{~J}$ was lower than that for ULV $\leq 10 \mathrm{~J}(23 \%$ vs. $51 \%, \mathrm{P}<0.0001$ ). On analysis of single 15-J ULV test only at the peak of T-wave, VF was not induced in 79 of 91 patients, but 4 of these had high DFT.
\end{abstract}

Conclusions: The 15-J ULV test with 3 coupling intervals could correctly identify high-DFT patients and reduce the necessity for VF induction at defibrillator implantation. (Circ J 2013; 77: 2490-2496)

Key Words: Defibrillation; Defibrillation threshold; Implantable cardioverter defibrillator; Upper limit of vulnerability; Ventricular fibrillation

$\mathbf{I}$ mplantable cardioverter defibrillators (ICDs) are established as the optimal approach to prevent sudden cardiac death due to malignant ventricular arrhythmias. ${ }^{1-3}$ And cardiac resynchronization therapy combined with ICD (CRTD) has been indicated in patients with advanced heart failure associated with electrical and/or mechanical dyssynchrony. ${ }^{4-7}$ At the time of implantation, an ICD generally has to be tested to ensure that it can reliably sense ventricular fibrillation (VF) and deliver a direct-current shock with an acceptable safety margin to terminate the VF once or twice. This test, however, requires repetitive VF induction and thus has potentially life-threatening complications. ${ }^{8-11}$ Therefore, defibrillation testing with minimal risk is required.

The upper limit of vulnerability (ULV) is the weakest shock strength at which VF can no longer be induced when a shock is delivered during the cardiac vulnerable period. A close correlation between the ULV and the defibrillation threshold (DFT) has been validated in some studies of humans and other animals, ${ }^{12-14}$ and the safety and reliability of ICD implantation and programming of the lower first-shock strength using vulnerability testing without VF induction as a surrogate for DFT assessment has been demonstrated. ${ }^{15-21}$

The present study evaluated the usefulness of vulnerability testing to confirm acceptable DFT at the time of ICD implantation and to predict high DFT $(>20 \mathrm{~J})$. Optimal strength and number of shocks at ULV testing to predict high DFT without inducing VF were assessed.

Received January 29, 2013; revised manuscript received May 8, 2013; accepted June 11, 2013; released online July 20, 2013 Time for primary review: 36 days

Division of Cardiovascular Medicine, Department of Internal Medicine, Kobe University Graduate School of Medicine, Kobe, Japan

Mailing address: Akihiro Yoshida, MD, Division of Cardiovascular Medicine, Department of Internal Medicine, Kobe University Graduate School of Medicine, Kusunoki-Cho 7-5-2, Chuoh-Ku, Kobe 650-0017, Japan. E-mail: ayoshida@med.kobe-u.ac.jp or kfuku@med. kobe-u.ac.jp

ISSN-1346-9843 doi:10.1253/circj.CJ-13-0149

All rights are reserved to the Japanese Circulation Society. For permissions, please e-mail: cj@j-circ.or.jp 


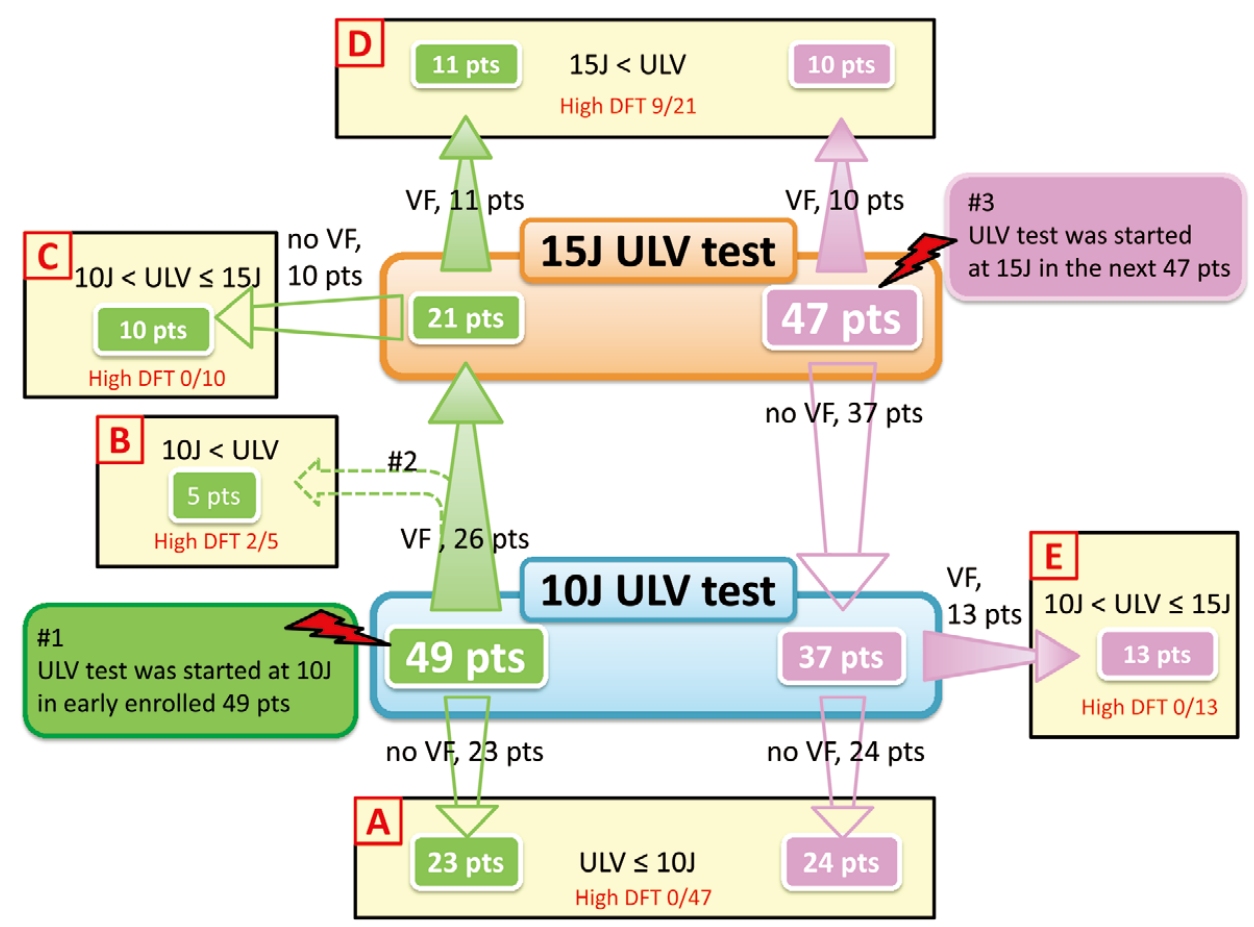

Figure. Protocol for vulnerability and defibrillation test, and patient flow. (\#1) The vulnerability testing was started at 10J in the early enrolled 49 patients. VF was induced at $10 \mathrm{~J}$ in 26 patients (53\%; ULV >10 J) and not induced in 23 (47\%; ULV $\leq 10 \mathrm{~J})$. Among the 26 patients in whom VF was induced at 10-J ULV test, 21 of them underwent testing at 15J. Among these 21 patients, the 15-J vulnerability test induced VF in 11 patients (ULV $>15 \mathrm{~J}$ ) and $(\mathbf{C})$ did not induce VF in 10 patients $(10 \mathrm{~J}<\mathrm{ULV} \leq 15 \mathrm{~J})$. But (\#2, B) 15-J ULV test could not be done in 5 patients because of their unstable hemodynamics (hypotension or bradycardia) after VF defibrillation. (\#3) The ULV test was started at $15 \mathrm{~J}$ in the next 47 patients. VF was induced at $15 \mathrm{~J}$ in 10 patients $(21 \%$; ULV $>15 \mathrm{~J})$ and not induced in 37 (79\%; ULV $\leq 15 \mathrm{~J})$. The 10-J ULV test was done in the 37 patients with ULV $\leq 15 \mathrm{~J}$. VF was induced at (E) $10 \mathrm{~J}$ ULV in 13 patients $(35 \% ; 10 \mathrm{~J}<U L V \leq 15 \mathrm{~J})$ and VF was not induced in 24 patients (65\%; ULV $\leq 10 \mathrm{~J})$. Accordingly, in all of the 96 patients, (A) ULV $\leq 10 \mathrm{~J}$ was confirmed in 47 patients (49\%) and (B-E) ULV >10 J was confirmed in 49 patients (51\%). With regard to the ULV $\leq$ or $>15 \mathrm{~J}$, it was able to be decided in 91 patients. Of these, (A,C,E) ULV $\leq 15 \mathrm{~J}$ was confirmed in 70 patients (77\%) and (D) ULV >15J was confirmed in 21 patients (23\%). DFT, defibrillation threshold; Pts, patients; ULV, upper limit of vulnerability; VF, ventricular fibrillation. High DFT defined as $>20 \mathrm{~J}$

\section{Methods}

\section{Patients}

The subjects consisted of 96 patients ( 80 men, 16 women) undergoing implantation of an ICD/CRTD for the first time $(n=75)$ or as a replacement $(n=21)$ between March 2006 and November 2008. All patients underwent transthoracic echocardiography and routine laboratory testing, and all patients gave written informed consent before the device implantation.

\section{Device Implantation}

Patients were anesthetized with propofol or midazolam. Heart rhythm, $\mathrm{O}_{2}$ saturation, and continuous systemic blood pressure were monitored throughout all procedures. Disposable adhesive defibrillator pads were applied to the patients and attached to the external defibrillator for rescue cardioversion. Various defibrillator models were implanted. The defibrillator system was inserted through a left pectoral incision in all patients except for 3 who had a history of device infection $(n=1)$ and dialysis vascular access $(n=2)$. The tip of the ventricular shock lead was placed at or near the right ventricular apex if the $R$ wave was $\geq 5 \mathrm{mV}$ and the pacing threshold was $\leq 1.5 \mathrm{mV}$ at $0.5 \mathrm{~ms}$. Before defibrillator replacement, previously implanted shock leads were con- firmed to satisfy all the aforementioned criteria.

\section{Determination of Vulnerable Period}

During right ventricular pacing with a cycle length (CL) of $500 \mathrm{~ms}$ via the shock lead, the latest peak of the monophasic Twave was determined on standard 12-lead electrocardiography (ECG) displayed at $100 \mathrm{~mm} / \mathrm{s}$, and the interval from the stimulus to the latest peak of the T-wave (St-T peak interval) was measured. A limb-lead ECG was substituted if a 12-lead ECG was unavailable. The vulnerable period was determined from -20 to $+20 \mathrm{~ms}$ of the latest T peak, as described. ${ }^{15,22}$

\section{Definition of Ventricular Fibrillation}

Arrhythmias with CL $<280 \mathrm{~ms}$ with polymorphic and irregular ( $\triangle \mathrm{CL}>40 \mathrm{~ms}$ ) QRS complexes on surface ECG were classified as VF. Arrhythmias with monomorphic QRS complexes or CL $>280 \mathrm{~ms}$ were defined as ventricular tachycardia and excluded from the VF count. ${ }^{23}$

\section{Vulnerability and Defibrillation Testing}

Vulnerability testing was started at $10 \mathrm{~J}$ in the early enrolled 49 patients (Figure). The first $10 \mathrm{~J} \mathrm{~T}$-wave shock was delivered at the St-T peak interval after 8 right ventricular pacing beats with 


\begin{tabular}{lc|}
\hline \multicolumn{1}{c|}{ Table 1. Patient Characteristics } & \\
Variable & Outcome $(\mathbf{n}=96)$ \\
Age (years) & $63 \pm 14$ \\
M/F & $80 / 16$ \\
Underlying heart disease & \\
Ischemic cardiomyopathy & 39 \\
Non-ischemic cardiomyopathy & 41 \\
No structural heart disease & 16 \\
LVEF (\%) & $43 \pm 18$ \\
CRTD & 15 \\
Indication for defibrillator & \\
VF or cardiac arrest & 32 \\
VT & 48 \\
Non-sustained VT & 16 \\
Anti-arrhythmic drugs & 22 \\
Amiodarone & 15 \\
Sotalol & 7 \\
Class I & 4 \\
$\beta$-blockers & 70 \\
ACEl or ARB & 70 \\
\hline
\end{tabular}

Data given as mean $\pm \mathrm{SD}$ or $\mathrm{n}$. ACEI, angiotensin-converting enzyme inhibitor; ARB, angiotensin receptor blocker; CRTD, cardiac resynchronization therapy defibrillator; LVEF, left ventricular ejection fraction; VF, ventricular fibrillation; VT, ventricular tachycardia.

$\mathrm{CL}=500 \mathrm{~ms}$. If $\mathrm{VF}$ was not induced, a subsequent $10-\mathrm{J}$ shock was delivered at $-20 \mathrm{~ms}$ and $+20 \mathrm{~ms}$ to the St-T peak interval. If three $10-\mathrm{J}$ shocks with different timing did not induce VF, we determined that the ULV was $\leq 10 \mathrm{~J}$, and then subsequent shocks were decreased to 5 or $1 \mathrm{~J}$, and delivered in the same sequence until $\mathrm{VF}$ was induced. If VF was not induced by a series of induction shocks, VF was induced by alternative methods such as a high frequency burst for defibrillation testing.

Vulnerability was tested at 1-min intervals between shocks. If VF was induced, defibrillation was tested. Five minutes after VF defibrillation, the energy of the next ULV test shock was increased to $15 \mathrm{~J}$ and delivered in the same manner. If VF was also induced at $15 \mathrm{~J}$, it was determined that the ULV was $>15 \mathrm{~J}$. If VF was not induced by any $15-\mathrm{J}$ T-wave shocks, the ULV was determined as $\leq 15 \mathrm{~J}$.

The aim of this study was to estimate the DFT without inducing VF. The first 10-J T-wave shocks, however, induced VF in many patients $(53 \%)$ using this protocol. Therefore, we changed the first induction shock to $15 \mathrm{~J}$ in the next 47 patients (Figure). The protocol for the 15-J shocks was the same as that described for the 10-J shocks.

During defibrillation testing for induced VF, we evaluated only whether DFT was $\leq 20 \mathrm{~J}$ or not. The first defibrillation shock was set at $20 \mathrm{~J}$ and the second was set at the maximum delivered energy of each device. If the first 20-J shock could not defibrillate (DFT $>20 \mathrm{~J}$ ), the first shock was increased by $5 \mathrm{~J}$ until VF was defibrillated in subsequent defibrillation tests. We defined DFT as the smallest shock strength that defibrillated twice.

In both vulnerability and defibrillation testing, shock energy was unified as delivered energy. Therefore, the programmed (charged) energy was adjusted to obtain the required delivered energy for the Guidant defibrillator (delivered energy of 10, 15 and $20 \mathrm{~J}$ was equivalent to charged energy of 11,17 and $23 \mathrm{~J}$, respectively).

If a patient became hemodynamically unstable or fell into VF several times during these tests, vulnerability and defibrillation testing were stopped according to the decision of the operator.

\section{Statistical Analysis}

Baseline characteristics are given as mean \pm SD and were analyzed for statistical significance using paired t-test for continuous variables and Fisher's exact test for categorical variables. $\mathrm{P}<0.05$ was considered statistically significant.

\section{Results}

Table 1 lists the patient characteristics. Anti-arrhythmic drugs (amiodarone, $\mathrm{n}=15$; sotalol, $\mathrm{n}=7$; mexiletine, $\mathrm{n}=2$; pirmenol, $\mathrm{n}=1$; procainamide, $\mathrm{n}=1$ ) were given to 26 patients and $\beta$-blockers were given to $70(73 \%)$ at the time of the procedure.

\section{Device Implantation}

At the time of ICD implantation, the mean $\mathrm{R}$ wave amplitude on the ventricular sensing lead was $12.6 \pm 5.11 \mathrm{mV}$ (range, 5.0$29.1 \mathrm{mV}$ ). The stimulation threshold was $<1.5 \mathrm{~V}$ at $0.5 \mathrm{~ms}$ in all patients. Dual-chamber ICDs and CRTDs were implanted in 73 and 15 patients, respectively. No major perioperative complications developed that required additional procedures.

\section{Vulnerable Period}

The mean St-T peak interval was $351 \pm 35 \mathrm{~ms}$. No visible T-wave alternans developed at right ventricular pacing at $500 \mathrm{~ms}$ CL.

\section{Vulnerability and Defibrillation Testing}

The ULV test was started at $10 \mathrm{~J}$ in the early enrolled 49 patients (Figure). VF was induced at $10 \mathrm{~J}$ in 26 patients $(53 \%$; $\mathrm{ULV}>10 \mathrm{~J})$ and not induced in $23(47 \%$; ULV $\leq 10 \mathrm{~J})$. Among the 26 patients in whom VF was induced at 10-J ULV test, 21 of them underwent testing at $15 \mathrm{~J}$. Among these 21 patients, the $15-\mathrm{J}$ ULV test induced VF in 11 patients (ULV $>15 \mathrm{~J}$ ) and did not induce VF in 10 patients $(10 \mathrm{~J}<\mathrm{ULV} \leq 15 \mathrm{~J}$; Figure $\mathrm{C})$. But the $15-\mathrm{J}$ ULV test could not be done in 5 patients because of unstable hemodynamics (hypotension or bradycardia) after VF defibrillation. Interestingly, 2 of these 5 patients had high DFT (Figure B).

The ULV test was started at $15 \mathrm{~J}$ in the next 47 patients (Figure). VF was induced by 15-J ULV test in 10 (21\%; ULV $>15 \mathrm{~J})$ and not induced in 37 (79\%; ULV $\leq 15 \mathrm{~J})$. The ULV test at $10 \mathrm{~J}$ was done in the 37 patients with $\mathrm{ULV} \leq 15 \mathrm{~J}$. VF was induced by $10 \mathrm{~J}$ ULV in 13 patients $(35 \% ; 10 \mathrm{~J}<\mathrm{ULV} \leq 15 \mathrm{~J}$; Figure $\mathbf{E})$ and VF was not induced in 24 patients $(65 \%$; ULV $\leq 10 \mathrm{~J})$.

Among the 47 patients without VF induction on the 10-J ULV test, 39 patients underwent the 5-J ULV test and VF was induced in 28 patients $(5 \mathrm{~J}<\mathrm{ULV} \leq 10 \mathrm{~J})$. The $1-\mathrm{J}$ ULV test was done in 19 patients (11 patients without VF induction by the 5-J ULV test and 8 patients who did not undergo the 5-J ULV test) and VF was induced in 17 patients $(1 \mathrm{~J}<\mathrm{ULV} \leq 5 \mathrm{~J}$ in 10 patients and $1 \mathrm{~J}<\mathrm{ULV} \leq 10 \mathrm{~J}$ in 7 patients). In the remaining 2 patients, high frequency burst pacing was required for inducing VF.

\section{ULV and High DFT}

In all of the 96 patients, ULV $\leq 10 \mathrm{~J}$ was confirmed in 47 patients (49\%; Figure A) and ULV >10 J was confirmed in 49 patients (51\%; Figure B-E). In total, 11 patients $(11 \%)$ had high DFT $(>20 \mathrm{~J})$ and ULV was $>10 \mathrm{~J}$ in all of these 11 patients. In 38 of 49 patients with ULV $>10$ J, however, DFT was $<20$ J (acceptable DFT). The sensitivity, specificity, positive predictive value (PPV) and negative predictive value (NPV) of ULV $>10 \mathrm{~J}$ for predicting high DFT were $100 \%, 55 \%, 22 \%$ and $100 \%$, respectively (Table 2).

Because 15-J ULV test could not be done in 5 patients (Figure B), the decision as to whether ULV was $\leq 15 \mathrm{~J}$ or $>15 \mathrm{~J}$ 


\begin{tabular}{|c|c|c|c|c|c|c|c|}
\hline & $\begin{array}{c}\mathrm{DFT} \leq 20 \mathrm{~J} \\
\text { (n) }\end{array}$ & $\begin{array}{c}\text { DFT }>20 \mathrm{~J} \\
\text { (n) }\end{array}$ & $\begin{array}{c}\text { Total } \\
\text { (n) }\end{array}$ & $\begin{array}{l}\text { SS } \\
(\%)\end{array}$ & $\begin{array}{l}\text { SP } \\
(\%)\end{array}$ & $\begin{array}{l}\text { PPV } \\
(\%)\end{array}$ & $\begin{array}{l}\text { NPV } \\
(\%)\end{array}$ \\
\hline$U L V \leq 10 \mathrm{~J}$ & 47 & 0 & 47 & & & & \\
\hline ULV $>10 \mathrm{~J}$ & 38 & 11 & 49 & 100 & 55 & 22 & 100 \\
\hline Total & 85 & 11 & 96 & & & & \\
\hline$U L V \leq 15 \mathrm{~J}$ & 70 & 0 & 70 & & & & \\
\hline ULV $>15 \mathrm{~J}$ & 12 & 9 & 21 & 100 & 85 & 43 & 100 \\
\hline Total & 82 & 9 & $91^{\dagger}$ & & & & \\
\hline
\end{tabular}

${ }^{\dagger}$ Five patients could not undergo ULV testing at 15J. DFT, defibrillation threshold; NPV, negative predictive value; PPV, positive predictive value; SP, specificity; SS, sensitivity; ULV, upper limit of vulnerability.

\begin{tabular}{|c|c|c|c|c|c|c|c|}
\hline & $\begin{array}{c}\text { DFT } \leq 20 \mathrm{~J} \\
(\mathrm{n})\end{array}$ & $\begin{array}{c}\text { DFT > }>20 \mathrm{~J} \\
\text { (n) }\end{array}$ & $\begin{array}{l}\text { Total } \\
\text { (n) }\end{array}$ & $\begin{array}{l}\text { SS } \\
(\%)\end{array}$ & $\begin{array}{l}\text { SP } \\
(\%)\end{array}$ & $\begin{array}{l}\text { PPV } \\
(\%)\end{array}$ & $\begin{array}{l}\text { NPV } \\
(\%)\end{array}$ \\
\hline \multicolumn{8}{|c|}{ 10-J ULV test with $3 \mathrm{Cl}$} \\
\hline No VF & 47 & 0 & 47 & & & & \\
\hline VF & 31 & 8 & 39 & 100 & 66 & 20 & 100 \\
\hline Total & 78 & 8 & 86 & & & & \\
\hline \multicolumn{8}{|c|}{ 15-J ULV test with $3 \mathrm{Cl}$} \\
\hline No VF & 47 & 0 & 47 & & & & \\
\hline VF & 12 & 9 & 21 & 100 & 80 & 42 & 100 \\
\hline Total & 59 & 9 & 68 & & & & \\
\hline \multicolumn{8}{|c|}{ 15-J ULV test with $1 \mathrm{Cl}$} \\
\hline No VF & 52 & 4 & 56 & & & & \\
\hline VF & 7 & 5 & 12 & 55 & 88 & 42 & 93 \\
\hline Total & 59 & 9 & 68 & & & & \\
\hline
\end{tabular}

$\mathrm{Cl}$, coupling interval. Other abbreviations see in Tables 1, 2 .

\begin{tabular}{|c|c|c|c|c|c|c|}
\hline Variables & $\begin{array}{c}U L V \leq 10 J \\
(n=47)\end{array}$ & $\begin{array}{c}U L V>10 J \\
(n=49)\end{array}$ & P-value & $\begin{array}{c}U L V \leq 15 J^{\dagger} \\
(n=70)\end{array}$ & $\begin{array}{c}U L V>15 J^{\dagger} \\
(n=21)\end{array}$ & P-value \\
\hline Age (years) & $63 \pm 14$ & $62 \pm 14$ & 0.93 & $62 \pm 15$ & $64 \pm 11$ & 0.50 \\
\hline $\mathrm{M} / \mathrm{F}$ & $39 / 8$ & $41 / 8$ & $>0.99$ & $58 / 12$ & $18 / 3$ & $>0.99$ \\
\hline \multicolumn{7}{|l|}{ Underlying heart disease } \\
\hline Ischemic cardiomyopathy & 23 & 16 & 0.14 & 29 & 8 & $>0.99$ \\
\hline Non-ischemic cardiomyopathy & 16 & 25 & 0.10 & 29 & 9 & $>0.99$ \\
\hline No structural heart disease & 8 & 8 & $>0.99$ & 12 & 4 & $>0.99$ \\
\hline $\operatorname{LVEF}(\%)$ & $43 \pm 17$ & $43 \pm 18$ & 0.88 & $45 \pm 18$ & $38 \pm 17$ & 0.10 \\
\hline LVDd (mm) & $57 \pm 9$ & $55 \pm 14$ & 0.38 & $55 \pm 10$ & $57 \pm 16$ & 0.51 \\
\hline LVDs (mm) & $44 \pm 12$ & $42 \pm 17$ & 0.51 & $42 \pm 13$ & $45 \pm 19$ & 0.35 \\
\hline CRTD & 4 & 11 & 0.09 & 8 & 5 & $>0.99$ \\
\hline Anti-arrhythmic drugs & 14 & 12 & 0.65 & 20 & 6 & 0.59 \\
\hline$\beta$-blockers & 36 & 34 & 0.49 & 53 & 13 & 0.27 \\
\hline ACEI or ARB & 37 & 33 & 0.25 & 51 & 14 & 0.17 \\
\hline St-T peak interval (ms) & $349 \pm 34$ & $353 \pm 35$ & 0.58 & $348 \pm 33$ & $353 \pm 37$ & 0.55 \\
\hline DFT $\geq 20 \mathrm{~J}$ & 0 & 11 & 0.0005 & 0 & 9 & $<0.0001$ \\
\hline
\end{tabular}

Data given as mean \pm SD or $n$. tFive patients could not undergo ULV testing at $15 \mathrm{~J}$. LVDd, left ventricular end-diastolic dimension; LVDs, LV end-systolic dimension; St-T, stimulus-T wave. Other abbreviations see in Tables 1, 2.

was made in 91 patients. ULV $\leq 15 \mathrm{~J}$ was confirmed in 70 patients (77\%; Figure A,C,E) and ULV $>15 \mathrm{~J}$ was confirmed in 21 patients (23\%; Figure D). A high DFT was not found in patients with ULV $\leq 15 \mathrm{~J}$, whereas 9 of 21 patients with ULV $>15 \mathrm{~J}$ had a high DFT. The sensitivity, specificity, PPV and NPV of ULV > 15 J for predicting high DFT were $100 \%, 85 \%$, $43 \%$ and $100 \%$, respectively (Table 2).
The rate of VF inducibility to confirm ULV $\leq 15 \mathrm{~J}$ was lower than that for ULV $\leq 10 \mathrm{~J}$ ( $23 \%$ vs. $51 \%, \mathrm{P}<0.0001)$.

\section{0-J ULV vs. 15-J ULV Test}

A total of 86 patients underwent 10-J ULV test and VF was induced in 39 patients (45\%; Table 3). And 68 patients underwent $15-\mathrm{J}$ ULV test and VF was induced in 21 patients (31\%). The 


\begin{tabular}{|c|c|c|c|}
\hline Variables & $\begin{array}{c}\text { DFT } \leq 20 \mathrm{~J} \\
(\mathrm{n}=85)\end{array}$ & $\begin{array}{c}\text { DFT >20J } \\
(n=11)\end{array}$ & P-value \\
\hline Age (years) & $62 \pm 14$ & $66 \pm 11$ & 0.35 \\
\hline$M / F$ & $72 / 13$ & $8 / 3$ & 0.39 \\
\hline \multicolumn{4}{|l|}{ Underlying heart disease } \\
\hline Ischemic cardiomyopathy & 36 & 3 & 0.52 \\
\hline Non-ischemic cardiomyopathy & 33 & 8 & 0.05 \\
\hline No structural heart disease & 16 & 0 & 0.20 \\
\hline LVEF (\%) & $44 \pm 18$ & $33 \pm 17$ & 0.05 \\
\hline LVDd (mm) & $55 \pm 11$ & $63 \pm 16$ & 0.05 \\
\hline LVDs (mm) & $42 \pm 14$ & $51 \pm 19$ & 0.07 \\
\hline CRTD & 12 & 3 & 0.37 \\
\hline Anti-arrhythmic drugs & 23 & 3 & $>0.99$ \\
\hline$\beta$-blockers & 62 & 8 & $>0.99$ \\
\hline ACEI or ARB & 60 & 10 & 0.28 \\
\hline St-T peak interval (ms) & $349 \pm 34$ & $367 \pm 39$ & 0.10 \\
\hline
\end{tabular}

Data given as mean \pm SD or $n$. Abbreviations see in Tables 1, 2, 4.

sensitivity and NPV of VF inducibility for both the 10-J and 15-J ULV test for predicting high DFT were $100 \%$. Importantly, the specificity and PPV of VF inducibility for the 15-J ULV test were higher than those for the 10-J ULV test (Table 3). Furthermore, the rate of VF inducibility for the 15-J ULV test was lower than that for the 10-J ULV test ( $31 \%$ vs. $45 \%, \mathrm{P}=0.07)$.

\section{Patient Characteristics and ULV}

Patient characteristics did not differ significantly between ULV $\leq 10 \mathrm{~J}$ and $>10 \mathrm{~J}$, and ULV $\leq 15 \mathrm{~J}$ and $>15 \mathrm{~J}$. Patients with high DFT, however, were more often identified with ULV $>10 \mathrm{~J}$ than $\leq 10 \mathrm{~J}(\mathrm{P}=0.0005)$, and with $\mathrm{ULV}>15 \mathrm{~J}$ than $\leq 15 \mathrm{~J}(\mathrm{P}<0.0001$; Table 4).

\section{Optimal Number of Shocks at ULV Test}

We delivered 3 shocks within the vulnerable period $(-20,0$, $+20 \mathrm{~ms}$ to the peak of T-wave wave). The effectiveness of the 15-J ULV test with single shock only at the peak of the T-wave was analyzed. VF was induced by single shock at $15 \mathrm{~J}$ in 12 of 68 patients, and a high DFT was found in 5 (42\%) of these 12 patients. Among 56 patients without VF induction by the single 15-J ULV test, 4 patients (7.1\%) had a high DFT. The sensitivity, specificity, PPV and NPV of VF inducibility by single 15-J shock for predicting a high DFT were 55\%, 88\%, $42 \%$ and $93 \%$, respectively (Table 3 ). The sensitivity of single 15-J ULV test was considerably lower than that for the 15-J ULV test with 3 coupling intervals, and the NPV of single ULV test was $<100 \%$.

\section{Defibrillation Testing and High DFT}

The ICD immediately sensed VF in all patients during defibrillation testing, and the $\mathrm{R}$ wave was not under-sensed during VF even when sensitivity was sufficiently blunted. Induced VF could not be terminated by a first rescue 20-J shock in 11 patients (high DFT). System modification such as repositioning of the shock lead or changing of the direction of the shock wave was required in 4 of them to achieve an adequate DFT safety margin (DFT $\leq$ maximum device output energy-10J).

Prevalence of non-ischemic cardiomyopathy $(\mathrm{P}=0.05)$, lower left ventricular $(\mathrm{LV})$ ejection fraction $(\mathrm{P}=0.05)$, larger $\mathrm{LV}$ enddiastolic and end-systolic dimension $(\mathrm{P}=0.05$ and 0.07 , respectively) were more common in patients with high DFT but the differences did not reach significance (Table 5).

\section{Number of Induction Shocks and Procedure Duration}

The mean number of induction T-wave shocks was $5.7 \pm 2.3$; the total duration of the test was $14.2 \pm 2.4 \mathrm{~min}$ and the amount of time required to confirm effective defibrillation energy (twice) or acceptable ULV $(\leq 10$ or $\leq 15 \mathrm{~J})$ was $6.2 \pm 4.7 \mathrm{~min}$ for all patients.

\section{Complications}

Neither major complications nor prolonged unstable hemodynamics requiring inotropic agents or mechanical support were encountered in this series.

\section{Discussion}

The major findings can be summarized as follows. The ULV test at 10 and $15 \mathrm{~J}$ is a reliable method to identify patients with an acceptable DFT $(\leq 20 \mathrm{~J})$. In patients with acceptable DFT, the rate of VF inducibility by 15-J ULV test was significantly lower than that for the 10-J ULV test. Single ULV test at the peak of T-wave sometimes underestimated the ULV. Baseline patient characteristics could not predict high DFT, but ULV could.

Based on these findings, the 15-J ULV test with 3 coupling intervals $(-20,0$, and $+20 \mathrm{~ms}$ to the peak of T-wave) was thought to be a reliable method to identify high-DFT patents without inducing VF.

\section{Previous Reports}

To date, a close correlation between the ULV and DFT at the time of defibrillator implantation has been reported. ${ }^{12-14}$ These findings suggest that vulnerability testing can be safely and reliably substituted for conventional defibrillation testing. Regarding device implantation without induction of VF, the 15-J ULV test was more feasible compared with the 10-J ULV test. Hwang et al showed that the ULV was $\leq 20 \mathrm{~J}$ in $75 \%$ of 60 patients, and all of them had DFT $\leq 20$ J. ${ }^{24}$ Swerdlow et al showed that inductionless implantation is feasible in $>80 \%$ of patients based on vulnerability testing at $15 \mathrm{~J} .^{25}$

The ASSURE study compared vulnerability safety margin testing vs. defibrillation safety margin test with a single VF induction/defibrillation; ${ }^{15}$ 14-J vulnerability testing with 3 coupling intervals was carried out in 420 patients. VF was not induced in 322 patients $(76.7 \%)$ and defibrillation was achieved with 21-J shocks in 317 of them (98.4\%). Among the 98 patients $(23.3 \%)$ in whom VF was induced at $14-\mathrm{J}$ vulnerability testing, $21(21.4 \%)$ had DFT $>21 \mathrm{~J}$. The predictive value of VF inducibility for detecting high DFT in the present study was comparatively higher than that of ASSURE. The ICD device produced by Guidant was used in the ASSURE study. In the Guidant ICD system, the programmed energy of $14 \mathrm{~J}$ is converted into $12 \mathrm{~J}$ at shock delivery and the programmed energy of $21 \mathrm{~J}$ is converted into $18 \mathrm{~J}$ at shock delivery. Vulnerability test at " $12 \mathrm{~J}$ " in the ASSURE study seems to be a reason for the relatively lower predictive value of VF inducibility for detecting high DFT.

\section{Determination of Vulnerable Period and Underestimation of ULV}

The vulnerable zone was defined as a combination of coupling intervals and the strength of the T-wave shock. It is shown as a 2-D, diamond-shaped space defined by the coupling interval on the abscissa and shock strength on the ordinate. ${ }^{26}$ Swerdlow et al investigated the timing of the peak of the human vulnerable zone using right ventricular pacing at a CL of $500 \mathrm{~ms}$ and showed that the peak of the human vulnerable zone is narrow and includes a median of only two 20 -ms intervals. ${ }^{13}$ They re- 
ported that a $20-\mathrm{ms}$ difference could cause underestimation of the ULV, and the most reliable method to define the ULV is to scan the vulnerable period of the T-wave shock within a window of $40 \mathrm{~ms}$ (in 20-ms steps) before and after the T-wave peak. We determined the vulnerable period as a window of $20 \mathrm{~ms}$ before and after the T-wave peak. Although this method might underestimate the ULV, none of the patients with either ULV $\leq 10 \mathrm{~J}$ or $\leq 15 \mathrm{~J}$ had a high DFT.

A single T-wave shock underestimates the actual ULV in the present study. In the TULIP study vulnerability test was done at a single coupling interval on the T-wave of the ECG lead II. ${ }^{16}$ Although this simplified method reduced the number of required induction shocks and the procedure time, the accuracy of measuring ULV might be reduced.

A T-wave shock was delivered after ventricular pacing at a CL of $400 \mathrm{~ms}$ in the TULIP study. ${ }^{16}$ Most of the ULV studies used a basic CL of $500 \mathrm{~ms}$. Pacing CL might affect the width of the vulnerable period. Furthermore, a shortened pacing CL might cause beat-to-beat instability of repolarization such as $\mathrm{T}$-wave alternans.

\section{Inducibility of VF During Vulnerability Testing}

All the patients in whom VF was not induced by ULV test at both $10 \mathrm{~J}$ and $15 \mathrm{~J}$ had an acceptable DFT $(\leq 20 \mathrm{~J})$. And in all the patients with high DFT, VF was induced by both 10-J and 15-J ULV test. The 10-J and 15-J ULV test, however, induced VF in 31 and in 12 patients with acceptable DFT, respectively. The higher delivered energy at ULV test might lower VF inducibility in patients without a high DFT, but it might involve a risk of missing a high DFT. Further detailed prospective study to identify the ideal energy at ULV test is required.

Avoidance of VF induction at defibrillator implantation is required in patients with severe heart failure. Unfortunately in these patients, VF is likely to be induced even by the vulnerability test. Patients with more advanced heart failure were more likely to have high DFT in the ASSURE study. ${ }^{15}$ In contrast, the parameters of cardiac function such as LV ejection fraction and LV dimensions did not predict high DFT in the present study, but ULV could predict high DFT. Although several studies have attempted to identify the predictive factors associated with high DFT, ${ }^{27-31}$ it has been difficult to identify high-DFT patients without vulnerability or defibrillation testing.

\section{Study Limitations}

The present study had some limitations. Shock energy other than 10 or $15 \mathrm{~J}$ might be optimal in vulnerability testing. Some patients might have a lower limit of vulnerability $>10 \mathrm{~J}$ or $>15 \mathrm{~J}$. This likelihood, however, seems to be vanishingly small because none of the patients without VF induced on 10-J ULV testing had high DFT.

Without inducing VF, R-wave sensing during induced VF cannot be ensured. But in patients with sinus rhythm R-wave sensing amplitude $>5 \mathrm{mV}$, R-wave sensing during VF was thought to be almost always reliable, and critical delay in detecting VF was not observed in previous reports. ${ }^{21}$

Determination of the vulnerable period using 12-lead ECG is essential for the ULV test. This process requires extra time and adequate experience. Recently, the usefulness of automated vulnerability test for predicting high DFT has been reported. ${ }^{21}$ The vulnerable period that was automatically calculated by their software was well correlated with the St-T peak interval. The 18-J vulnerability test at 4 coupling intervals could correctly detect high-DFT patients with $19 \%$ of VF inducibility. The automated ULV test may be able to increase the utility of the ULV test in clinical practice.
Multiple induction shocks might increase DFT. More than 10 multiple induction shocks were sometimes required in the present study. If these induction shocks increased the DFT, DFT might be overestimated, but we did not identify a significant dissociation between ULV and DFT.

\section{Clinical Implications}

At the time of defibrillator implantation, the 15-J ULV test with 3 coupling intervals $(-20,0$, and $+20 \mathrm{~ms}$ to the peak of T-wave) was thought to be a reliable method to detect high DFT. The vulnerable period should be determined as $-20,0$ and $+20 \mathrm{~ms}$ to the St-T peak interval using multi-lead ECG during pacing at a $\mathrm{CL}$ of $500 \mathrm{~ms}$. If any of the $3 \mathrm{~T}$-wave shocks cannot induce VF, no further induction is required and implantation without VF induction can be completed.

Although the ULV test can decrease the necessity for VF induction, VF tends to be induced by the ULV test in patients with depressed LV function, in whom VF induction is hoped to be avoided. Accordingly, we have considered the indication of the ULV test as follows. For the secondary prevention of sudden cardiac death, ULV test should be done. And, if VF is induced, defibrillation safety margin should be ensured, and system revision should be done if needed. For primary prevention, the ULV test might be avoided in patients with severely depressed LV function. Importantly, the necessity of ULV test should be decided on a patient-by-patient basis.

\section{Conclusion}

The ULV test at 10 and $15 \mathrm{~J}$ with 3 coupling intervals (-20, 0 , and $+20 \mathrm{~ms}$ to the peak of T-wave) is a reliable method to identify patients with an acceptable DFT $(\leq 20 \mathrm{~J})$. Baseline patient characteristics did not predict high-DFT patients, but ULV could detect high-DFT patients. For the purpose of defibrillator implantation without inducing VF, the 15-J ULV test was more feasible than the 10-J test.

\section{Disclosures}

This work was not financially supported.

\section{References}

1. Moss AJ, Hall WJ, Cannom DS, Daubert JP, Higgins SL, Klein H, et al. Improved survival with an implanted defibrillator in patients with coronary disease at high risk for ventricular arrhythmia. Multicenter Automatic Defibrillator Implantation Trial Investigators. N Engl J Med 1996; 335: $1933-1940$.

2. Connolly SJ, Hallstrom AP, Cappato R, Schron EB, Kuck KH, Zipes $\mathrm{DP}$, et al. Meta-analysis of the implantable cardioverter defibrillator secondary prevention trials. AVID, CASH and CIDS studies. Antiarrhythmics vs Implantable Defibrillator study. Cardiac Arrest Study Hamburg. Canadian Implantable Defibrillator Study. Eur Heart J 2000; 21: $2071-2078$.

3. A comparison of antiarrhythmic-drug therapy with implantable defibrillators in patients resuscitated from near-fatal ventricular arrhythmias. The Antiarrhythmics versus Implantable Defibrillators (AVID) Investigators. N Engl J Med 1997; 337: 1576-1583.

4. Young JB, Abraham WT, Smith AL, Leon AR, Lieberman R, Wilkoff $\mathrm{B}$, et al. Combined cardiac resynchronization and implantable cardioversion defibrillation in advanced chronic heart failure: The MIRACLE ICD Trial. JAMA 2003; 289: 2685-2694.

5. Strik M, Ploux S, Vernooy K, Prinzen FW. Cardiac resynchronization therapy: Refocus on the electrical substrate. Circ J 2011; 75: 1297 1304.

6. Momomura S, Tsutsui H, Sugawara Y, Ito M, Mitsuhashi T, Fukamizu $\mathrm{S}$, et al. Clinical efficacy of cardiac resynchronization therapy with an implantable defibrillator in a Japanese population: Results of the MIRACLE-ICD outcome measured in Japanese indication (MOMIJI) study. Circ J 2012; 76: 1911-1919.

7. Tanaka H, Tatsumi K, Fujiwara S, Tsuji T, Kaneko A, Ryo K, et al. Effect of left ventricular dyssynchrony on cardiac sympathetic activ- 
ity in heart failure patients with wide QRS duration. Circ J 2012; 76: 382-389.

8. Swerdlow CD, Russo AM, Degroot PJ. The dilemma of ICD implant testing. Pacing Clin Electrophysiol 2007; 30: 675-700.

9. Frame R, Brodman R, Furman S, Kim SG, Roth J, Ferrick K, et al Clinical evaluation of the safety of repetitive intraoperative defibrillation threshold testing. Pacing Clin Electrophysiol 1992; 15: 870877.

10. Singer I, Lang D. Defibrillation threshold: Clinical utility and therapeutic implications. Pacing Clin Electrophysiol 1992; 15: 932-949.

11. Vlay SC. Defibrillation threshold testing: Necessary but evil? Am Heart J 1989; 117: 499-504

12. Chen PS, Feld GK, Kriett JM, Mower MM, Tarazi RY, Fleck RP, et al. Relation between upper limit of vulnerability and defibrillation threshold in humans. Circulation 1993; 88: 186-192.

13. Swerdlow CD, Martin DJ, Kass RM, Davie S, Mandel WJ, Gang ES, et al. The zone of vulnerability to T wave shocks in humans. $J$ Cardiovasc Electrophysiol 1997; 8: 145-154.

14. Chen PS, Shibata N, Dixon EG, Martin RO, Ideker RE. Comparison of the defibrillation threshold and the upper limit of ventricular vulnerability. Circulation 1986; 73: $1022-1028$.

15. Day JD, Doshi RN, Belott P, Birgersdotter-Green U, Behboodikhah $\mathrm{M}$, Ott $\mathrm{P}$, et al. Inductionless or limited shock testing is possible in most patients with implantable cardioverter-defibrillators/cardiac resynchronization therapy defibrillators: Results of the multicenter ASSURE Study (Arrhythmia Single Shock Defibrillation Threshold Testing Versus Upper Limit of Vulnerability: Risk Reduction Evaluation With Implantable Cardioverter-Defibrillator Implantations). Circulation 2007; 115: 2382-2389.

16. Lemke B, Lawo T, Zarse M, Lubinski A, Kreutzer U, Mueller J, et al Patient-tailored implantable cardioverter defibrillator testing using the upper limit of vulnerability: The TULIP protocol. Europace 2008; 10: 907-913.

17. Kirilmaz A, Dokumaci B, Uzun M, Kilicaslan F, Dinckal MH, Yucel $\mathrm{O}$, et al. Detection of the defibrillation threshold using the upper limit of vulnerability following defibrillator implantation. Pacing Clin Electrophysiol 2005; 28: 498-505.

18. Swerdlow CD, Shehata M, Chen PS. Using the upper limit of vulnerability to assess defibrillation efficacy at implantation of ICDs. Pacing Clin Electrophysiol 2007; 30: 258-270.

19. Green UB, Garg A, Al-Kandari F, Ungab G, Tone L, Feld GK. Successful implantation of cardiac defibrillators without induction of ventricular fibrillation using upper limit of vulnerability testing. J Interv Card Electrophysiol 2003; 8: 71-75.
20. Glikson M, Gurevitz OT, Trusty JM, Sharma V, Luria DM, Eldar M, et al. Upper limit of vulnerability determination during implantable cardioverter-defibrillator placement to minimize ventricular fibrillation inductions. Am J Cardiol 2004; 94: 1445-1449.

21. Birgersdotter-Green U, Ruetz LL, Anand K, Monir G, Abeyratne AI, Bailey JR, et al. Automated vulnerability testing identifies patients with inadequate defibrillation safety margin. Circ Arrhythm Electrophysiol 2012; 5: 1073-1080.

22. Swerdlow C, Shivkumar K, Zhang J. Determination of the upper limit of vulnerability using implantable cardioverter-defibrillator electrograms. Circulation 2003; 107: 3028-3033.

23. Zima E, Gergely M, Soos P, Geller LA, Nemes A, Acsady G, et al. The effect of induction method on defibrillation threshold and ventricular fibrillation cycle length. J Cardiovasc Electrophysiol 2006; 17: $377-381$.

24. Hwang C, Swerdlow CD, Kass RM, Gang ES, Mandel WJ, Peter CT, et al. Upper limit of vulnerability reliably predicts the defibrillation threshold in humans. Circulation 1994; 90: 2308-2314.

25. Swerdlow CD. Implantation of cardioverter defibrillators without induction of ventricular fibrillation. Circulation 2001; 103: 2159-2164.

26. Fabritz CL, Kirchhof PF, Behrens S, Zabel M, Franz MR. Myocardial vulnerability to $\mathrm{T}$ wave shocks: Relation to shock strength, shock coupling interval, and dispersion of ventricular repolarization. J Cardiovasc Electrophysiol 1996; 7: 231-242.

27. Russo AM, Sauer W, Gerstenfeld EP, Hsia HH, Lin D, Cooper JM, et al. Defibrillation threshold testing: Is it really necessary at the time of implantable cardioverter-defibrillator insertion? Heart Rhythm 2005; 2: 456-461.

28. Khalighi K, Daly B, Leino EV, Shorofsky SR, Kavesh NG, Peters $\mathrm{RW}$, et al. Clinical predictors of transvenous defibrillation energy requirements. Am J Cardiol 1997; 79: 150-153.

29. Hodgson DM, Olsovsky MR, Shorofsky SR, Daly B, Gold MR. Clinical predictors of defibrillation thresholds with an active pectoral pulse generator lead system. Pacing Clin Electrophysiol 2002; 25: 408-413.

30. Chapman PD, Sagar KB, Wetherbee JN, Troup PJ. Relationship of left ventricular mass to defibrillation threshold for the implantable defibrillator: A combined clinical and animal study. Am Heart J 1987; 114: $274-278$

31. Schwartzman D, Concato J, Ren JF, Callans DJ, Gottlieb CD, Preminger MW, et al. Factors associated with successful implantation of nonthoracotomy defibrillation lead systems. Am Heart J 1996; 131: $1127-1136$. 